\title{
ON THE ORIENTATION OF THE MAJOR AXES OF PLANETARY NEBULAE
}

\author{
V.P. Grinin and A. M. ZvereVA \\ (Crimean Astrophyscial Observatory, U.S.S.R.)
}

The orientation of the major axes of planetary nebulae was investigated and the results are given in Figure 1. The solid line gives the distribution of 132 planetary nebulae as a function of the angle $\Delta \phi$ between the major axis of the nebula and the galactic equator. It can be seen that the distribution is not uniform, and is not symmetrical about $\Delta \phi=0$. The dashed line gives the distribution of the same nebulae as a function of the angle between major axis of the nebula and the direction of the interstellar magnetic field, which is determined from the stellar polarization measurements of stars close to the individual nebulae. This distribution is less uniform than the first one but is symmetrical about $\Delta \phi=0$. These results lead to the conclusion that the orientation of planetary nebulae is partly determined by the interstellar magnetic field.

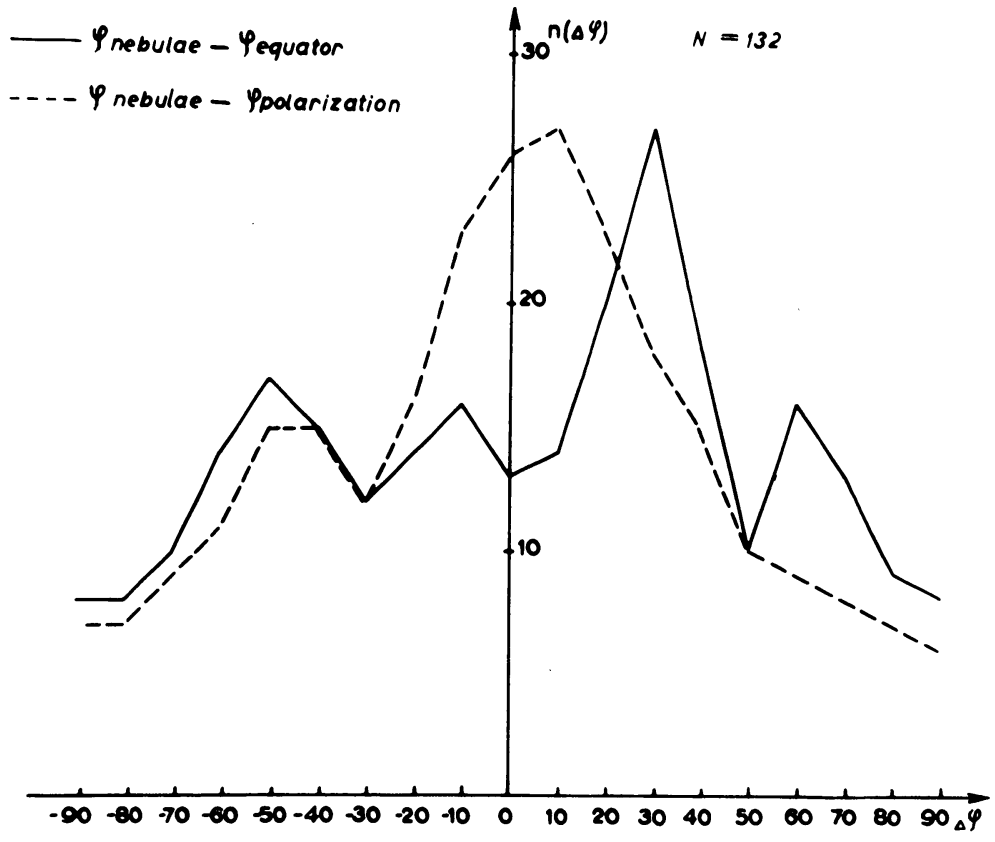

FIG. 1. 
In order to check this conclusion, the planetary nebulae were divided into two groups, with large and small galactic latitude $\left(b \gtrless 10^{\circ}\right)$. Then the above procedure was repeated, with the results given in Figure 2. It can be seen that the effect is stronger for nebulae with $b<10^{\circ}$, and is almost absent for nebulae which are far from the galactic equator. The conclusion that a correlation exists between the orientation of planetary nebulae and the interstellar magnetic field is thus confirmed.

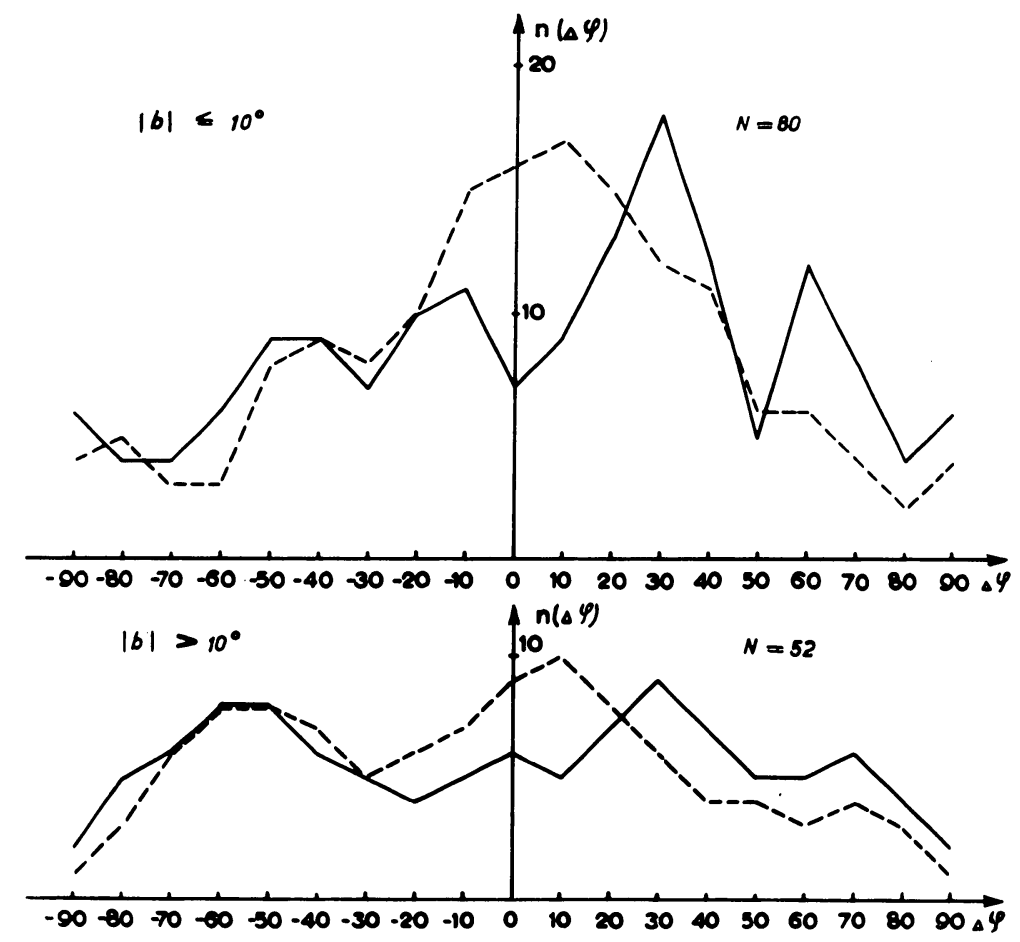

FIG. 2.

It is noted that the mean angle $\langle\Delta \phi\rangle$ between the major axis of the planetary nebulae and the galactic equator is the same as was found by Shain for the mean angle between the axes of elongated dark and emission nebulae and the galactic equator.

This contribution is to be published in full in Astrophysica (U.S.S.R.).

\section{DISCUSSION}

Gershberg: I should like to emphasize that Grinin and Zvereva's results show that there is no way to avoid the conclusion that the expansion of large planetary nebulae is influenced by the galactic magnetic field. It is known that the same phenomenon exists in diffuse nebulae. In diffuse nebulae, 
however, the density is lower than in planetary nebulae. However, the similarity in orientation implies that the ratio of magnetic pressure to gas pressure must be about the same in the two types of nebulae. Therefore the magnetic fields near planetary nebulae must be larger than the mean galactic field by a factor 10-30. It seems that such an intensified magnetic field can result from the compression of the galactic field by an expanding nebula. 